(2) Open Access Full Text Article

\title{
A comparative double-blind randomized study on the effectiveness of Duloxetine and Gabapentin on painful diabetic peripheral polyneuropathy
}

This article was published in the following Dove Press journal:

Drug Design, Development and Therapy

\section{Nastaran Majdinasab' \\ Hossein Kaveyani ${ }^{2}$ \\ Mojgan Azizi ${ }^{3}$}

'Musculoskeletal Rehabilitation Research Center, Jundishapur University of Medical Sciences, Ahvaz, Iran; ${ }^{2}$ Department of Neurology, Golestan Hospital, Ahvaz Jundishapur University of Medical Sciences, Ahvaz, Iran; ${ }^{3}$ Department of Pediatrics, Golestan Hospital, Ahvaz Jundishapur University of Medical Sciences, Ahvaz, Iran
Correspondence: Hossein Kaveyani Department of Neurology, Golestan Hospital, Ahvaz Jundishapur University of Medical Sciences, Ahvaz, Khuzestan, Iran Tel +98916 66I 2007

Fax +989166612007

Email drkaveiani@gmail.com
Background: The most common cause of polyneuropathy is diabetes mellitus. Neuropathic pain is seen in $26 \%$ of diabetic population. Therapeutic techniques for this disease can become challenging.

Method: This study was a prospective comparative double-blind randomized study which was conducted during an eight-week period. Totally, 104 painful diabetic peripheral polyneuropathy (PDPP) patients who had a minimum Visual Analog Scale (VAS) of 40 millimeters, received no pain-controlling medication, and had no other severe disease at its final stage were randomly assigned to two groups $(n=52)$ through the four block method. One group received Duloxetine and the other received Gabapentin. The effectiveness was measured through primary effectiveness (VAS scale) and secondary effectiveness (Sleep Interference Score, and Clinical Global Impression of Change (CGIC)). Medication compliance was assessed by enumerating the number of patients who refused treatment because of side effects. The Fisher's exact T-test and ANOVA were used for data analysis. This study was approved by the Ethics Committee of Jundishapur, University of Medical sciences Ahvaz, Iran, under reference number: IR.AJUMS.REC.1395.78. In addition, this study was registered and approved in the Iranian Registry of Clinical Trials (IRCT ID: IRCT20161023030455N2) (http://irct.ir/).

Results: VAS, Sleep Interference Score, and CGIC were significantly improved $(\mathrm{P}<0.05)$ through time in both groups, [For GBP: VAS $^{\text {Baseline }}=64 \pm 20.03$, VAS ${ }^{\text {week } 1}=55.32 \pm 18.76$, VAS $^{\text {week } 4}=44.68 \pm 15.82, \quad$ VAS $^{\text {week } 8}=39.43 \pm 14.32 ; \quad$ For $\quad$ DLX: $\quad$ VAS $^{\text {Base-line }}=62 \pm 21.18$, $\left.\mathrm{VAS}^{\text {week } 1}=58.76 \pm 20.37, \mathrm{VAS}^{\text {week } 4}=45.84 \pm 16.21, \mathrm{VAS}^{\text {week } 8}=36.78 \pm 15.62\right]$ while a significant difference between the two groups was not observed $(\mathrm{P}<0.05)$. However, such significant improvements were not observed in the Duloxetine group at the end of the first week $(\mathrm{P}=674)$. Improvement in Sleep Interference Score and CGIC were similar to the results for the VAS scale. Side effects in the Duloxetine group $(n=2)$ compared to the Gabapentin group $(n=9)$ were significantly less $(\mathrm{P}<0.001)$. As a result, medication acceptance in the Duloxetine group $(\mathrm{n}=47)$ was significantly better than the Gabapentin $(\mathrm{n}=41)$ group $(\mathrm{P}<0.001)$.

Conclusion: Both Duloxetine and Gabapentin are effective for the treatment of PDPP. On the one hand, Gabapentin shows the effect earlier while has more side effects. Conversely, Duloxetine has better medication compliance.

Trial registration: The method of this study was approved by the Ethics Committee of Jundishapur University of Medical Sciences, Ahvaz, Iran, under reference number: IR. AJUMS.REC.1395.78. In addition, this study was registered and approved in the Iranian Registry of Clinical Trials (IRCT ID: IRCT20161023030455N2) (http://irct.ir/).

Keywords: Duloxetine, Gabapentin, painful diabetic peripheral neuropathy 


\section{Introduction}

In Public Health Clinics, the most common cause of polyneuropathy is diabetes. The general symptoms of neuropathy vary and can range from minor dysesthesia to uncontrolled severe pain which can decrease life quality and the function of an individual. ${ }^{1-3}$ Diabetic peripheral neuropathy can be without symptoms. But in cases presenting symptoms, they can be in the form of positive ones like pain and prickling or negative ones like numbness and weakness. ${ }^{4,5}$ Peripheral neuropathy manifests itself in the form of symmetric distal neuropathy in most diabetic patients and its main symptoms are sensory and autonomic. This form of diabetic neuropathy is axonal and its progress depends on the length of the nerve fiber, ie, the longer the nerve fiber the sooner it will be engaged (the feet are engaged first). ${ }^{6}$ A form of neuropathy is seen among $60-70 \%$ of diabetic patients. Patients who have painful diabetic peripheral neuropathy feel severe pain, burning, dagger pain, and itching in their body. ${ }^{7-9}$

Diabetic neuropathic changes start with pain or disorders in the primary functions of the nervous system. ${ }^{10}$ Since neuropathic symptoms intensify at night, sleep disorders happen and this not only affects the patient's quality of life, but also worsens his/her diabetes. ${ }^{11}$

A mellitus diabetic patient who complains of foot or lower limb pain is normally diagnosed with painful diabetic peripheral neuropathy. These symptoms are so important. Patients who do not have proved diabetes but complain from such symptoms are recommended to take a two-hour oral glucose tolerance test. However, other diagnoses which can cause peripheral pain such as vitamin B12 deficiency or Osteoarthritis should be taken into consideration. ${ }^{12,13}$ A link between the intensity of disorder in glucose metabolism and neuropathy development has been shown in studies on patients who represented a series of disorders like the glucose tolerance test, ie, the effect of hyperglycemia in creating neuropathy in patients who showed disorders in their glucose tolerance test was proven. In accordance to this, patients recently diagnosed with diabetes neuropathy is milder. The engagement of small nerve fibers is the first detectable sign of the neuropathy. ${ }^{14}$

The basis of treatment and control of all diabetic complications is glucose control. However, in addition to glucose control, other medications and other non-pharmacological methods are used for controlling diabetic complications. In this case and for the treatment of painful diabetic peripheral neuropathy, prescription of antidepressant and anticonvulsant medication is suggested. ${ }^{15}$
As an anticonvulsant medication, Gabapentin is commonly used for treatment of neuropathic diabetes. ${ }^{1,16}$ Gabapentin acts like Pregabalin by connecting to the a-2d subunit of voltage gated calcium channels. ${ }^{17}$

Duloxetine is a norepinephrine serotonin reuptake inhibitor and used for the treatment of major depression. ${ }^{15}$ Duloxetine helps control pain through pain decreasing pathways. ${ }^{18}$ This medication was gradually approved by the FDA for the treatment of PDPP. ${ }^{19,20}$ A study on the comparative effectiveness of Duloxetine and Gabapentin (head to head) on the treatment of PDPP on Iranian patients does not exist. So, the current study compares the effectiveness and compliance of these two medications in patients who have this disease in order to provide better insight into its treatment.

\section{Method}

\section{Subjects}

The subjects of this clinical trial were selected from the outpatients of the Neurology and Endocrinology Clinic of Gloestan Hospital of Ahvaz, Iran, who met to the following criteria.

Inclusion criteria in the study:

1. The subjects were between 18 and 75 years old.

2. Subjects should be painful diabetic peripheral polyneuropathy from 1 month to 5 years and based on history, clinical examination (distal symmetric sensorimotor neuropathy as shown by pain, dysesthesias, numbness, sticking, lancinating, prickling, burning, aching, boring,impaired pin prick,temperature, or vibration sensation in both feet and absent or reduced ankle reflexes) or electrophysiology study.

3. Their HbAlc was lower than 10.

4. The time frame of their diabetes diagnosis was between 1 to 15 years.

5. Their VAS scale was at least 40 millimeters $(\mathrm{mm})$ out of $100 \mathrm{~mm}^{23}$

Exclusion criteria:

1. They were either using medication for painful diabetic peripheral neuropathy treatment or less than a week had passed from the discontinuation of their previous treatment.

2. They were banned from the use of Duloxetine or Gabapentin. 
3. They had severe illness in one of their vital organs such as their livers or kidneys (GFR less than $30 \mathrm{ml} /$ $\min$ ) or other organs.

4. They had amputation in their lower limb (even in a part of an organ). ${ }^{25,26,27}$

5. They showed symptoms of cognitive impairment (MMSE less than 25).

6 . They were pregnant or lactating.

7. They were alcohol abusers or drug addicts.

8. They had participated in another clinical trial in the past three months. ${ }^{27}$

The method of this study was approved by the Ethics Committee of Jundishapur, University of Medical Sciences, Ahvaz, Iran, under reference number: IR. AJUMS.REC.1395.78. In addition, this study was registered and approved in the Iranian Registry of Clinical Trials (IRCT ID: IRCT20161023030455N2) (http://irct.ir/).

\section{Procedure}

This clinical trial lasted for 8 weeks and it was prospective, double-blind, and randomized, and it involved parallel groups. Patients suitable for the study (based on the characteristics mentioned in the previous section) were divided into groups A (Gabapentin receivers) and B (Duloxetine receivers) by the four block randomized method (equalized four-blocks). A hundred and four patients were needed for this study ( 52 for each group). ${ }^{27}$

The medications of this study were first made similar to each other by a doctor who had no role in the collection and analysis of data and then sufficient amounts were packed into packets $\mathrm{A}$ and $\mathrm{B}$ and were given to the researcher. Before the commencement of the study, the side effects of the medications were explained to the patients and each patient randomly received one of the two medications used in the study.

Group A patients initially received 300 milligrams of oral Gabapentin a day and if they could tolerate the medication their received dose increased 300 milligrams every three days until it reached a daily dose of three 300 milligrams of oral Gabapentin (a daily maximum of 900 milligrams). Group B patients initially received 30 milligrams of Duloxetine single dose before sleep and if they could tolerate the medication for a week their daily dose was increased to a single 60 milligram dose of Duloxetine before sleep. The treatment period for both groups was eight weeks (20). The patients were assessed by a doctor who did not have information about the patients' medication type before treatment introduction and in weeks one, four, and eight.

The following factors were assessed in this study:

1. Primary effect: it is assessed by the VAS scale. This scale variates between 0 and $100 \mathrm{~mm}$ with zero indicating no pain and $100 \mathrm{~mm}$ indicating the worst possible pain. ${ }^{24}$ Before the introduction of the treatment, the VAS scale was assessed and then it was compared to the VAS scales assessed at weeks one, four, and eight. These comparisons were both intragroup, for assessing medication effectiveness and the effect of time on medication effectiveness, and intergroup, for comparing the effectiveness of the two medications.

2. Secondary effect: This was assessed by the Sleep Interference Score and the Clinical Global Impression of Change (CGIC). The Sleep Interference Score has 0 until 10 points and it is defined as the extent in which pain causes sleep disorders (zero indicates that pain causes no interference in sleep and 10 indicates that the patient cannot sleep because of pain). This factor was also assessed before the introduction of the treatment and also at weeks one, four, and eight and these assessments were compared just as the VAS assessments. CGIC assesses any changes in the patient's clinical condition and is graded on a seven-point scale. Each point indicates a specific clinical condition and it is defined as follows: 1 shows significant improvement; 2 shows major improvement; 3 shows minimal improvement; 4 shows no change; 5 shows minimal worsening; 6 shows major worsening; and 7 shows significant worsening of the clinical condition of the patient. This factor was assessed at weeks one, four, and eight and comparisons were made between them (according to the definition for CGIC, assessment of this factor before the introduction of the treatment is not meaningful). ${ }^{25,26}$

Primary and secondary effects (except CGIC) were assessed before treatment introduction and at the end of week one, four, and eight and in-group and between-group comparisons were made. ${ }^{25,26}$ Medication compliance was assessed through the number of patients who discontinued medication use because of its side effects. 


\section{Sample size and data analysis}

Subject number was determined as 104 according to Cang et al's study which also accounts for subject drop out. ${ }^{26}$

$$
\begin{gathered}
\mathrm{n}=[(\mathrm{z} 1-\alpha / 2+\mathrm{z} 1-\beta) 2(\mathrm{~s} 12+\mathrm{s} 22)] /(\mathrm{x} 1+\mathrm{x} 2) 2 \\
\mathrm{z} 1-\alpha / 2=1.96 \\
\mathrm{z} 1-\beta=0.84 \\
\mathrm{~s} 1=2.1 \sqrt{ } 27 \\
\mathrm{~s} 2=2.1 \sqrt{ } 27 \\
\mathrm{x} 1=16.3 \\
\mathrm{x} 2=22.3
\end{gathered}
$$

The patients were randomly assigned to two groups of 52 members through the four block method. Data description for qualitative variables was done through determining frequencies and percentiles, and calculation of mean and standard variation was used for the description of quantitative variables. Data analysis was performed through the use of Fisher's exact test, Chi-square, and analysis of variance (ANOVA). SPSS version 22 was used for data analysis.

\section{Ethics}

The method of this study was approved by the Ethics Committee of Jundi Shapour University of Medical Sciences, Ahvaz, Iran, under reference number: IR. AJUMS.REC.1395.78. In addition, this study was registered and approved in the Iranian Registry of
Clinical Trials (IRCT ID: IRCT20161023030455N2) (http://irct.ir/).

All patients were briefed on the procedure before the start of the study, and all participants have confirmed and signed the written Informed Consent Form. Although the medications used were known and they were commonly used for the treatment of certain diseases and symptoms and had no dangerous side effects, all patients were briefed on the common side effects and they were informed that any side effects caused by them will be treated by the research team. It was also explained to the patients that the medication they receive will be randomly assigned and no one would receive placebo. In addition, it was said to them that they can voluntarily enter and leave the study at any time they wish. The information obtained was considered as confidential and it was not given to another third party. It should also be mentioned that no costs were enforced on the patients in the study and their consent was obtained in accordance with the Helsinki Treaty.

\section{Results}

Hundred and ninety eight patients were screened in order to obtain a sample of 104. Ninety four patients did not have the specified characteristics and they were excluded for the following reasons: 11 patients did not have the required VAS scale, 41 patients used other pain-controlling medication, 25 patients had an HbA1c of more than 10, 7

\begin{tabular}{|c|c|c|c|}
\hline Characteristics & GBP (Mean \pm SD) & DLX (Mean \pm SD) & P-Value \\
\hline Age (years) & $60.7 \pm 5.66$ & $59.7 \pm 5.59$ & 0.224 \\
\hline \multicolumn{4}{|l|}{ Sex } \\
\hline Male & 21 & 24 & 0.356 \\
\hline Female & 31 & 28 & \\
\hline $\mathrm{HbA}, \mathrm{C}$ & $8.3 \pm 1.63$ & $7.8 \pm 1.96$ & 0.187 \\
\hline Duration of DM (y) & $7.86 \pm 4.28$ & $8.17 \pm 4.97$ & 0.784 \\
\hline Duration DPNP (y) & $3.58 \pm 1.86$ & $3.93 \pm 1.23$ & 0.435 \\
\hline Baseline VAS & $63 \pm 20.03$ & $61 \pm 21.18$ & 0.104 \\
\hline Baseline sleep interference score & $7.12 \pm 1.63$ & $6.86 \pm 1.57$ & 0.369 \\
\hline
\end{tabular}
patients had renal failure and 3 had heart failure, 1 patient had amputation on his/her lower limb, 2 patients had cognitive impairment, and 4 patients were drug abusers. The two groups were demographically and clinically similar (Table 1).

Table I Demographic and clinical information of patients before treatment introduction

Abbreviations: DM, Diabetes mellitus; DPNP, Diabetic peripheral neuropathic pain; DLX, Duloxetine; GBP, Gabapentin; VAS, Visual Analogue Scale. 
Forty two patients $(89.69 \%)$ in the Gabapentin group completed the study and seven patients (13.46\%) left the study because of side effects. Two patients (3.84\%), for reasons other than side effects, left the study because they did not want to continue their participation.

Forty seven patients $(90.38 \%)$ in the Duloxetine group completed the study and two patients (3.84\%) left the study because of side effects. Three patients, for reasons other than side effects, left the study because they did not want to continue their participation (one patient left the study because of immigration and two patients did not attend examination). A summary of the above information is presented in Graph 1.

At the end of Week four and Week eight, the VAS scale had improved significantly in both groups compared to pre-treatment introduction $(\mathrm{P}<0.05$, Table 2$)$. However, a significant difference in VAS was not observed between the two groups (Table 2). The VAS scale in the Gabapentin group showed significant improvement compared to before treatment at the end of Week one $(\mathrm{P}<0.05)$ and a significant decrease in VAS compared to before treatment was not observed for the Duloxetine group ( $\mathrm{P}=0.674)$ (Table 2). Also, decrease in VAS in the Gabapentin group compared to the Duloxetine group was only significant at the end of Week one $(\mathrm{P}=0.028)$ (Table 2). The VAS scale improved by the passage of time.

Improvement in Sleep Interference Score at the end of weeks one, four, and eight was similar to the results for the VAS scale (Table 3). A meaningful two-way relationship between time elapsed from the onset of treatment and Improvement in Sleep Interference Score was observed in both groups.

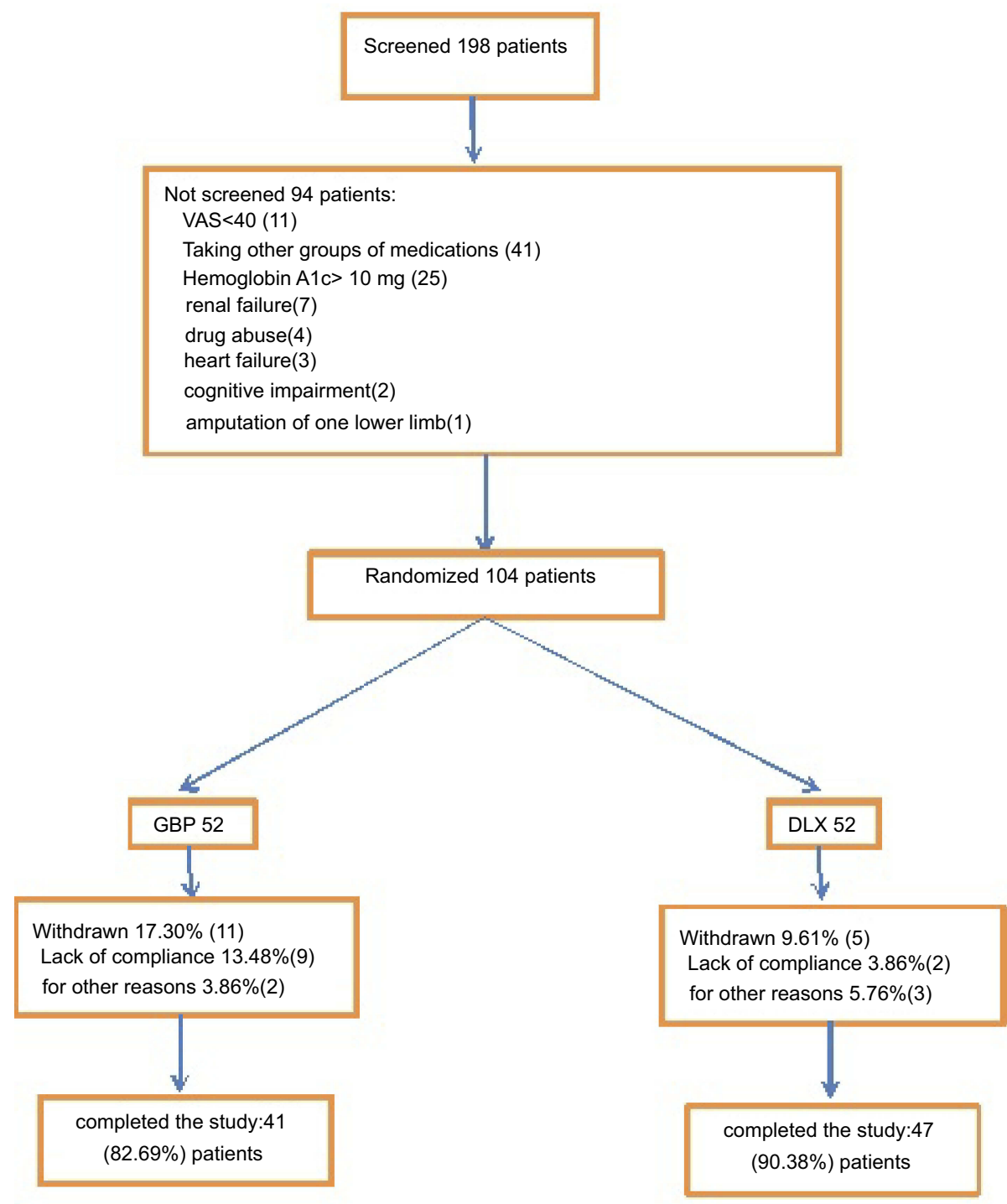

Graph I Profile of the randomized.

Abbreviations: DLX, Duloxetine; GBP, Gabapentin; VAS, Visual Analogue Scale. 
Table 2 Comparison of VAS scale before treatment and after I, 4, and 8 weeks

\begin{tabular}{|c|c|c|c|c|c|}
\hline \multicolumn{2}{|c|}{ Character } & \multirow{2}{*}{\multicolumn{2}{|c|}{ GBP (Mean \pm SD) }} & \multirow[t]{2}{*}{ DLX (Mean \pm SD) } & \multirow[t]{2}{*}{ P-Value } \\
\hline \multicolumn{2}{|c|}{ VAS Scale: } & & & & \\
\hline \multicolumn{2}{|c|}{ Pre-treatment (Base line) } & \multicolumn{2}{|l|}{$64 \pm 20.03$} & $62 \pm 21.18$ & \\
\hline \multicolumn{2}{|c|}{ After Week I } & \multicolumn{2}{|l|}{$55.32 \pm 18.76$} & $58.76 \pm 20.37$ & $\mathrm{P}=0.028$ \\
\hline \multicolumn{2}{|c|}{ After Week 4} & \multicolumn{2}{|l|}{$44.68 \pm 15.82$} & $45.84 \pm \mid 6.21$ & $P=0.607$ \\
\hline \multicolumn{2}{|c|}{ After Week 8} & \multicolumn{2}{|l|}{$39.43 \pm 14.32$} & $36.78 \pm 15.62$ & $\mathrm{P}=0.357$ \\
\hline P-Value & & $\begin{array}{l}\text { e VAS and after I weeks } \\
\text { e VAS and after } 4 \text { \& } 8 \text { weeks }\end{array}$ & $\begin{array}{l}P=0.000 \\
P<0.05\end{array}$ & $\begin{array}{l}P=0.674 \\
P<0.05\end{array}$ & \\
\hline
\end{tabular}

Abbreviations: DLX, Duloxetine; GBP, Gabapentin; VAS, Visual Analogue Scale.

Table 3 Comparison of sleep interference score before treatment and after I, 4, and 8 weeks

\begin{tabular}{|l|l|l|l|}
\hline Character & GBP (Mean \pm SD) & DLX (Mean \pm SD) & P-Value \\
\hline Sleep interference score: & & & \\
Pre-treatment (Base line) & $7.12 \pm 1.63$ & $6.86 \pm 1.57$ & \\
After Week I & $5.89 \pm 1.56$ & $6.64 \pm 1.74$ & $\mathrm{P}=0.003$ \\
After Week 4 & $4.68 \pm 1.82$ & $4.84 \pm 1.21$ & $\mathrm{P}=0.196$ \\
After Week 8 & $3.43 \pm 1.32$ & $2.78 \pm 1.62$ & $\mathrm{P}=0.081$ \\
P-Value & $\mathrm{P}<0.05$ & $\mathrm{P}<0.05$ & \\
\hline
\end{tabular}

Abbreviations: DLX, Duloxetine; GBP, Gabapentin.

The results for CGIC were similar to VAS, and the improvement in CGIC was meaningful $(\mathrm{P}<0.05)$ (Table 4). There was a meaningful effect between time elapse and CGIC improvement in both Gabapentin and Duloxetine groups $(\mathrm{P}<0.05)$. CGIC improvement in the Gabapentin group compared to the Duloxetine group was meaningful at the end of Week one, but this difference was not meaningful at the end of Weeks four and eight.

Side effects were significantly lower in the Duloxetine group (two patients) compared to the Gabapentin group (nine patients) $(\mathrm{P}<0.001)$. As a result, the compliance in the former group (47 patients) was significantly higher than the latter (41 patients) $(\mathrm{P}<00.01)$.

The most common side effects in the Gabapentin group were sleeplessness (five patients), imbalance (three patients), and fatigue (one patient). The most common side effects in the Duloxetine group were anxiety (one patient) and sleeplessness

Table 4 Comparison of CGIC in end of Weeks one, four, and eight after treatment between the Gabapentin and Duloxetine groups

\begin{tabular}{|l|l|l|l|}
\hline Characters & GBP & DLX & P-Value \\
\hline CGIC I & $3.31 \pm 1.04$ & $3.87 \pm 091$ & $\mathrm{P}=0.011$ \\
CGIC 4 & $2.96 \pm 0.48$ & $2.86 \pm 0.66$ & $\mathrm{P}=0.391$ \\
CGIC 8 & $2.04 \pm 0.37$ & $2.04 \pm 0.37$ & $\mathrm{P}=0.185$ \\
\hline
\end{tabular}

Abbreviations: DLX, Duloxetine; GBP, Gabapentin. (one patient). All side effects recovered after a short period of drug discontinuation, and as mentioned before, two patients in the Gabapentin group and three patients in the Duloxetine group left the study for reasons other than side effects such as immigration and family problems.

\section{Discussion}

The main treatment for all PDPP patients is maintaining glucose concentrations within the normal range. ${ }^{21}$ Consensus-based treatment guidelines recommended both pregabalin and duloxetine for managing PDPP patients as first-line medications. ${ }^{22}$

A limited number of studies have directly (head to head) compared the effectiveness of the two medications used for treating PDPP. ${ }^{23}$ This study showed that VAS significantly decreased in both groups and the two medications were both effective for treating the symptoms of painful diabetic peripheral polyneuropathy. VAS mean score in the end of Week one was significantly better in the Gabapentin group compared to the Duloxetine group. However, in the end of Weeks four and eight, all assessed criterions had significantly improved in both groups and no significant difference between the two groups was observed.

Medication compliance in the Duloxetine group was significantly better. On the basis of these findings, it can be said that patients who have PDPP recover faster 
by using Gabapentin if they could tolerate its side effects. A large placebo-control study showed that Gabapentin is effective in the treatment of painful diabetic peripheral neuropathy. ${ }^{1,15}$ A recent randomized, multi-center placebo-controlled study showed that Duloxetine was more effective than placebo in controlling PPP and it improved VAS mean score by $50 \%$ in $24 \mathrm{hrs}^{26}$ The results of the above studies are in line with the results of this study and show that both medications are effective in treating pain of the painful diabetic peripheral polyneuropathy. In addition, the results show that both medications are effective in improving Sleep Interference Score and CGIC. However, Gabapentin has a more rapid effect than Duloxetine, but this disappears at end of Weeks four and eight and the effect of both medications becomes similar with time elapsing.

Similar medication compliance for both Gabapentin and Duloxetine has been reported in previous studies. ${ }^{27}$ However, these studies were not done on Iranian patients, and the present study shows that the side effects of Gabapentin are significantly higher than Duloxetine for Iranians

\section{Conclusion}

Gabapentin and Duloxetine were equally effective in improving VAS, Sleep Interference Score, and CGIC in patients who had PDPP and the effectiveness of both medications was significantly meaningful. In addition, Duloxetine showed fewer side effects, but the effect of Gabapentin started faster. It can be concluded that for preventing side effects, Duloxetine can be used. However, in order to compensate for the late start of its effect, other pain-controlling medications such as tricyclic antidepressants, nonsteroidal anti-inflammatory drugs, or Gabapentin in lower doses should be used in the first week. After Week one, these medications should be discontinued and only Duloxetine should be used. Evidently, this proposal needs further investigation and further studies can be conducted for more investigation into this issue.

\section{Ethical approval}

All procedures performed in study involving human participants were in accordance with the ethical standards of the national research committee and with the 2008 Helsinki declaration and its later amendments or comparable ethical standards [Ethical Code: IR.AJUMS. REC.1395.78].

\section{Acknowledgments}

This study was funded by Ahvaz Jundishapur University of Medical Sciences (grant number IR.AJUMS.REC.1395.78).

\section{Disclosure}

Dr Nastaran Majdinasab, Dr Hossein Kaveyani, and Dr Mojgan Azizi have received research grants from Ahvaz Jundishapur University of Medical Sciences (grant number IR.AJUMS.REC.1395.78). The authors report no other conflicts of interest in this work.

\section{References}

1. Backonja M, Beydoun A, Edwards KR, et al. Gabapentin for the symptomatic treatment of painful neuropathy in patients with diabetes mellitus: a randomized controlled trial. Jama. 1998;280(21):1831-1836.

2. Galer BS, Gianas A, Jensen MP. Painful diabetic polyneuropathy: epidemiology, pain description, and quality of life. Diabetes Res Clin Pract. 2000;47(2):123-128.

3. Boulton AJ, Vinik AI, Arezzo JC, et al. Diabetic neuropathies a statement by the American Diabetes Association. Diabetes Care. 2005;28(4):956-962.

4. Davies M, Brophy S, Williams R, Taylor A. The prevalence, severity, and impact of painful diabetic peripheral neuropathy in type 2 diabetes. Diabetes Care. 2006;29(7):1518-1522. doi:10.2337/dc05-2228

5. Dyck PJ, Albers JW, Andersen H, Arezzo JC, Biessels GJ, Bril V, et al. Diabetic polyneuropathies: update on research definition, diagnostic criteria and estimation of severity. Diabetes Metab Res Rev. 2011;27(7):620-628.

6. Said G. Diabetic neuropathy - a review. Nat Clin Pract Neurol. 2007;3(6):331-340. doi:10.1038/ncpneuro0504

7. Vinik AI. Diabetic neuropathy: pathogenesis and therapy. Am J Med. 1999;107(2):17-26. doi:10.1016/S0002-9343(99)00009-1

8. Schmader KE. Epidemiology and impact on quality of life of postherpetic neuralgia and painful diabetic neuropathy. Clin J Pain. 2002;18(6):350-354.

9. Argoff CE, Cole BE, Fishbain DA, Irving GA, ed. Diabetic peripheral neuropathic pain: clinical and quality-of-life issues. Mayo Clinic Proceedings; 2006: Elsevier. doi:10.1016/S0025-6196(11)61474-2

10. Young M, Boulton A, MacLeod A, Williams D, Sonksen P. A multicentre study of the prevalence of diabetic peripheral neuropathy in the United Kingdom hospital clinic population. Diabetologia. 1993;36(2):150-154.

11. Gottlieb DJ, Punjabi NM, Newman AB, et al. Association of sleep time with diabetes mellitus and impaired glucose tolerance. Arch Intern Med. 2005;165(8):863-867. doi:10.1001/archinte.165.8.863

12. King SA. Diabetic peripheral neuropathic pain: effective management. Consultant. 2008;48(11):825.

13. Rutkove SB. A 52-year-old woman with disabling peripheral neuropathy: review of diabetic polyneuropathy. JAMA. 2009;302 (13):1451-1458. doi:10.1001/jama.2009.1377

14. Sumner C, Sheth S, Griffin J, Cornblath D, Polydefkis M. The spectrum of neuropathy in diabetes and impaired glucose tolerance. Neurology. 2003;60(1):108-111.

15. Chong MS, Hester J. Diabetic painful neuropathy. Drugs. 2007;67 (4):569-585. doi:10.2165/00003495-200767040-00006

16. Backonja M, Glanzman RL. Gabapentin dosing for neuropathic pain: evidence from randomized, placebo-controlled clinical trials. Clin Ther. 2003;25(1):81-104.

17. Sills GJ. The mechanisms of action of gabapentin and pregabalin. Curr Opin Pharmacol. 2006;6(1):108-113. doi:10.1016/j. coph.2005.11.003 
18. Robinson MJ, Ahl J, Meyers AL, McCarberg BH, Iyengar S. Management of painful symptoms with duloxetine: a review of the efficacy in pre-clinical and clinical studies. Curr Drug Ther. 2011;6 (2):121-136. doi:10.2174/157488511795304903

19. Wong DT, Bymaster FP, Mayle DA, Reid LR, Krushinski JH, Robertson DW. LY248686, a new inhibitor of serotonin and norepinephrine uptake. Neuropsychopharmacology. 1993;8(1):23-33. doi:10.1038/npp.1993.4

20. Bymaster FP, Dreshfield-Ahmad LJ, Threlkeld PG, et al. Comparative affinity of duloxetine and venlafaxine for serotonin and norepinephrine transporters in vitro and in vivo, human serotonin receptor subtypes, and other neuronal receptors. Neuropsychopharmacology. 2001;25(6):871-880. doi:10.1016/ S0893-133X(01)00298-6

21. Amthor KF, Dahl-Jorgensen K, Berg TJ, et al. The effect of 8 years of strict glycaemic control on peripheral nerve function in IDDM patients: the Oslo Study. Diabetologia. 1994;37:579-584.

22. Argoff CE, Backonja MM, Belgrade MJ, et al. Consensus guidelines: treatment planning and options. Mayo Clin Proc. 2006;81(Suppl 4): S12-S25. doi:10.1016/S0025-6196(11)61475-4
23. Devi P, Madhu K, Ganapathy B, Sarma G, John L, Kulkarni C. Evaluation of efficacy and safety of gabapentin, duloxetine, and pregabalin in patients with painful diabetic peripheral neuropathy. Indian J Pharmacol. 2012;44(1):51. doi:10.4103/0253-7613.91867

24. Tanenberg RJ, Irving GA, Risser RC, et al., ed. Duloxetine, pregabalin, and duloxetine plus gabapentin for diabetic peripheral neuropathic pain management in patients with inadequate pain response to gabapentin: an open-label, randomized, noninferiority comparison. Mayo Clinic Proceedings; 2011: Elsevier. doi:10.4065/mcp.2010.0681

25. Boyle J, Eriksson ME, Gribble L, et al. Randomized, placebo-controlled comparison of amitriptyline, duloxetine, and pregabalin in patients with chronic diabetic peripheral neuropathic pain impact on pain, polysomnographic sleep, daytime functioning, and quality of life. Diabetes Care. 2012;35(12):2451-2458. doi:10.2337/dc12-0656

26. Goldstein DJ, Lu Y, Detke MJ, Lee TC, Iyengar S. Duloxetine vs. placebo in patients with painful diabetic neuropathy. Pain. 2005;116 (1):109-118. doi:10.1016/j.pain.2005.03.029

27. Quilici S, Chancellor J, Löthgren M, et al. Meta-analysis of duloxetine vs. pregabalin and gabapentin in the treatment of diabetic peripheral neuropathic pain. BMC Neurol. 2009;9(1):1. doi:10.1186/1471-2377-9-6
Drug Design, Development and Therapy

\section{Publish your work in this journal}

Drug Design, Development and Therapy is an international, peerreviewed open-access journal that spans the spectrum of drug design and development through to clinical applications. Clinical outcomes, patient safety, and programs for the development and effective, safe, and sustained use of medicines are a feature of the journal, which has also

\section{Dovepress}

been accepted for indexing on PubMed Central. The manuscrip management system is completely online and includes a very quick and fair peer-review system, which is all easy to use. Visit http://www. dovepress.com/testimonials.php to read real quotes from published authors. 